\title{
Proposition de formulations pour le calcul du coefficient de réflexion sur une structure imperméable à talus
}

\author{
Maria Cristina Garcia Govea ${ }^{1}$, Daniel Levacher ${ }^{2}$, Daniel Caminade ${ }^{3}$
}

${ }^{1}$ Docteur, Instituto Mexicano del Petroleo, Eje Central Lazaro Cardenas No. 152, Col. San Bartolo Atepehuacan, Edificio 1, $2^{\circ}$ piso, CP 07730, Mexico D.F. Mexico, Téléphone: +52 55 30036088, Fax: +52 55 30036087,E-mail :mcgarcia@imp.mx

${ }^{2}$ Professeur, Université de Caen, UMR 6143 CNRS Morphodynamique Continentale et Côtière, 24 rue des Tilleuls, 14000 Caen, France, E-mail :daniel.levacher@geos.unicaen.fr Téléphone:33+231565709,

${ }^{3}$ Maître de Conférences, ISA-BTP, Laboratoire des Sciences Appliquées au Génie Civil, LASAGEC, Allée du Parc Montaury, 64600 Anglet, France, , E-mail : daniel.caminade@univ-pau.fr Téléphone : $33+559577002$

\section{Résumé}

Ce travail concerne une étude expérimentale en canal menée sur une digue imperméable et submersible soumise à l'action d'une houle régulière. L'ensemble des essais a été effectué dans l'un des canaux à houle du Centre de Géomorphologie de l'Université de Caen. Le modèle de digue construit à cet effet, avait pour caractéristiques géométriques les valeurs suivantes: $0.40 \mathrm{~m}$ de hauteur, $0.20 \mathrm{~m}$ de largeur en crête et des pentes talutées à $4 / 3$. Un des objectifs était de déterminer la réflexion de la houle en amont de la structure. Il nous a paru intéressant, à partir des données expérimentales recueillies lors de ces essais, de proposer des formulations semi-empiriques relatives au calcul du coefficient de réflexion sur les digues à talus.

\begin{abstract}
This paper concerns the influence of monochromatic waves on an impervious breakwater. Wave interaction with a slope breakwater is studied by means of a physical model. Experimental works were carried out at the University of Caen, on the research unit "Morphodynamique Continentale et Cotiere". One of the main objectives of this work is to determine the wave reflection as a function of hydraulic and structural parameters. Breakwater model was built with a geometry of $0.40 \mathrm{~m}$ high, $0.20 \mathrm{~m}$ wide at crest and a slope of $3 / 4$. The results of experimental model tests are used to formulate semi-empirical equations to predict wave reflection coefficient.
\end{abstract}




\section{Introduction}

La réflexion se présente lorsque la houle atteint une paroi en formant un système d'ondes stationnaires appelé clapotis. L'amplitude de ces ondes est sensiblement égale au double de celle de la houle incidente; leur longueur d'onde et leur période restant identiques à celles de la houle incidente.

La quantité d'énergie réfléchie par une plage ou par une structure marine peut être déterminée par le coefficient de réflexion $\left(\mathrm{K}_{\mathrm{r}}\right)$ qui se présente généralement, comme une fonction du nombre d'Iribarren $\xi_{0}$.

$$
\xi_{0}=\tan \alpha / \sqrt{H_{0} / L_{0}}
$$

Ainsi, de nombreux auteurs ou ouvrages donnent des courbes pour la fonction $f\left(\xi_{0}\right)$ et fournissent des valeurs du coefficient $K_{r}$ pour les pentes douces, les plages de sable et les digues en enrochements.

Seelig (1983) propose la formule suivante applicable aux digues submersibles:

$$
K_{r}=\frac{a \xi_{0}^{2}}{b+\xi_{0}^{2}}
$$

Postman (cité par Pilarczyk, 1996), propose :

$$
K_{r}=0,14 \xi_{p}^{0,73} \text { avec un écart type } \sigma\left(K_{r}\right)=0,055
$$

Szmytkiewicz (cité par Pilarczyk, 1996) du laboratoire IBW PAN en Pologne, arrive à la relation suivante:

$$
K_{r}=0,17 \xi_{p}^{0,95}
$$

\section{Dispositif expérimental}

L'étude expérimentale (Garcia-Govea 2000a) a été realisée dans un canal à houle, dont les caractéristiques géométriques sont les suivantes: $22 \mathrm{~m}$ de longueur totale, $0.80 \mathrm{~m}$ de largeur et une hauteur utile de $0.80 \mathrm{~m}$ (voir figure 1). A fin d'éviter les réflexions multiples qui viennent perturber la zone d'essais un amortisseur de houle a été disposé en bout de canal (Garcia-Govea et al 2000b) .

Le modèle de digue imperméable construit à cet effet, avait pour caractéristiques géométriques les valeurs suivantes : $0.40 \mathrm{~m}$ de hauteur, $0.20 \mathrm{~m}$ de largeur en crête et des pentes talutées à $4 / 3$. 


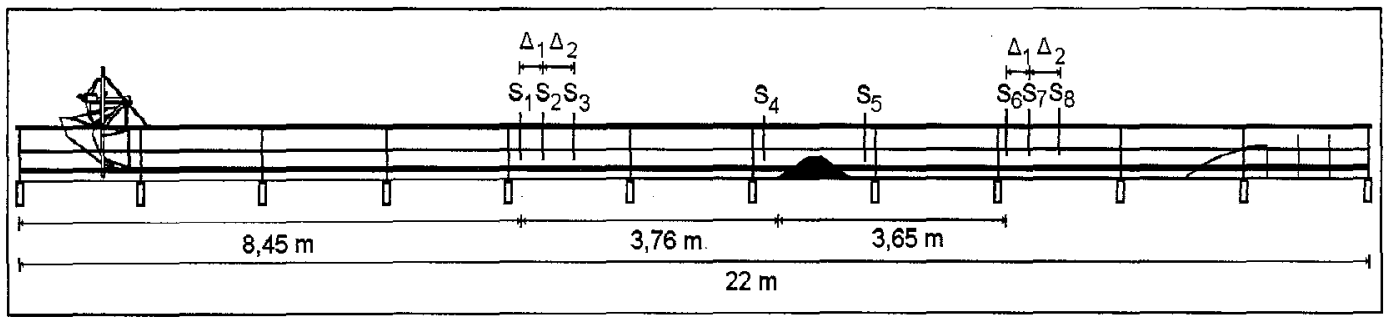

Figure 1 :Vue schématique du canal - Wave flume view

\subsection{Instrumentation}

Les caractéristiques de la houle sont enregistrées à l'aide de sondes résistives reliées à un moniteur de houle. La figure 2 montre sous forme schématique la chaîne d'acquisition employée pour la mesure simultanée de la surface libre en 8 points du canal. Les trois premières sondes sont installées dans la partie amont de la structure pour déterminer avec précision la houle incidente et la réflexion de la digue, les sondes 4 et 5 mesurent la surface libre au voisinage de la structure et les trois dernières permettent de mesurer la transmission de la houle.L'ensemble des traitements effectués sur les signaux de surface libre a été réalisé à l'aide de programmes développés par CHEVALIER (2000) et par ROUSSET (1998), en utilisant certaines routines de calculs du logiciel scientifique MATLAB ${ }^{\circ}$. Nous rappelons que tous nos essais ont été menés en houle régulière. Au cours des essais, nous avons utilisé la méthode de Mansard et Funke (1980) pour la séparation de la houle.

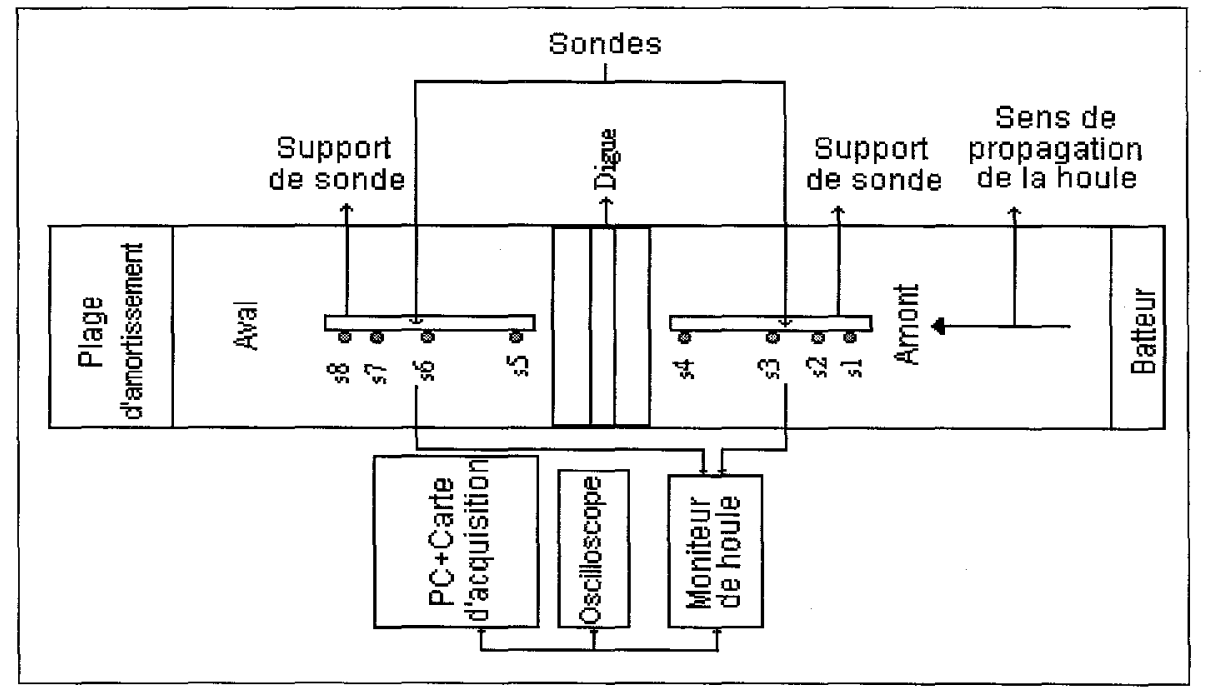

Figure 2 : Mesures et chaîne d'acquisition - Position of wave gauges

\section{Paramètres hydrauliques}

Les paramètres hydrauliques choisis pour cette étude sont présentés dans la tableau 1. 


\begin{tabular}{|c|c|c|}
\hline $\begin{array}{c}\text { Niveau d'eau } \\
\mathrm{h}(\mathrm{m})\end{array}$ & $\begin{array}{c}\text { Période de la houle } \\
\mathrm{T}(\mathrm{s})\end{array}$ & $\begin{array}{c}\text { Hauteur de la houle } \\
\mathrm{H}(\mathrm{m})\end{array}$ \\
\hline 0,35 & 1,00 & $0,020-0,096(6$ valeurs* $)$ \\
0,35 & 1,15 & $0,016-0,116(7$ valeurs*) \\
0,35 & 1,30 & $0,015-0,104(8$ valeurs*) \\
0,35 & 1,50 & $0,022-0,109(7$ valeurs* $)$ \\
0,35 & 1,80 & $0,023-0,089(5$ valeurs*) \\
0,35 & 2,20 & $0,032-0,078(5$ valeurs* $)$ \\
\hline
\end{tabular}

Tableau 1 : Paramètres hydrauliques - Hydraulic parameters

*nombre de hauteurs de houle mesurées

\section{Résultats}

Comme aucun modèle mathématique s'avère fiable pour calculer la réflexion de la houle, les modèles de laboratoire restent les moyens d'études les plus appropriés pour l'estimation du paramètre de réflexion.

La valeur du coefficient de réflexion décroît lorsque une grande quantité de l'énergie est transmisse par franchissement de la digue. Nous avons constaté, compte tenu de l'étendue des périodes étudiées, que la période est un paramètre qui ne peut pas être négligée et qui a une influence considérable sur le coefficient de réflexion. Le coefficient de réflexion décroît au fur et à mesure que la période diminue.

\subsection{Influence de la cambrure de la houle et de la période sur le coefficient de réflexion}

Le coefficient de réflexion décroît lorsque la cambrure $(\mathrm{Hi} / \mathrm{L})$ augmente. Les valeurs minimales de $\mathrm{Kr}$ correspondent à une houle de courte période et de forte amplitude, tandis que les valeurs maximales correspondent à une houle de grande période et de faible amplitude. Ceci signifie qu'une houle longue est mieux réfléchie qu'une courte, (voir figure 3).

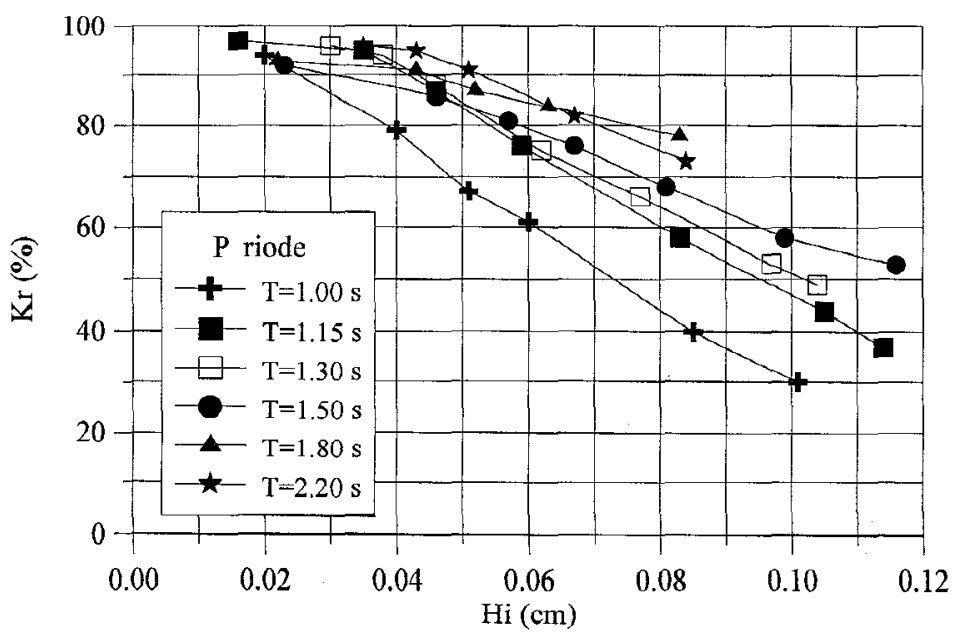

Figure 3 : Influence de la période de la houle sur le coefficient de réflexion.

Wave period influence over reflection coefficient 
4.2 Comparaison des résultats expérimentaux avec les différentes théories et formulations émises

Les coefficients de réflexion expérimentaux ont été confrontés avec la formulation donnée par Seelig (équation 2), dont les coefficients $a=1$ et $b=5,5$ sont proposés pour une structure à pente lisse.

La figure 4, présente la comparaison de résultats expérimentaux et théoriques pour chacune de périodes testées. Les valeurs de $\mathrm{Kr}$ sont voisines de $100 \%$ tant que l'amplitude n'a pas atteint une valeur suffisante pour que la houle franchisse la digue. On note que pour les faibles amplitudes, les valeurs expérimentales sont plus élevées que les valeurs théoriques, mais ceci s'inverse pour les fortes amplitudes. Pour cette profondeur relative, il existe un assez bon accord avec les résultats, principalement pour les périodes voisines de $\mathrm{T}=1,80 \mathrm{~s}$.
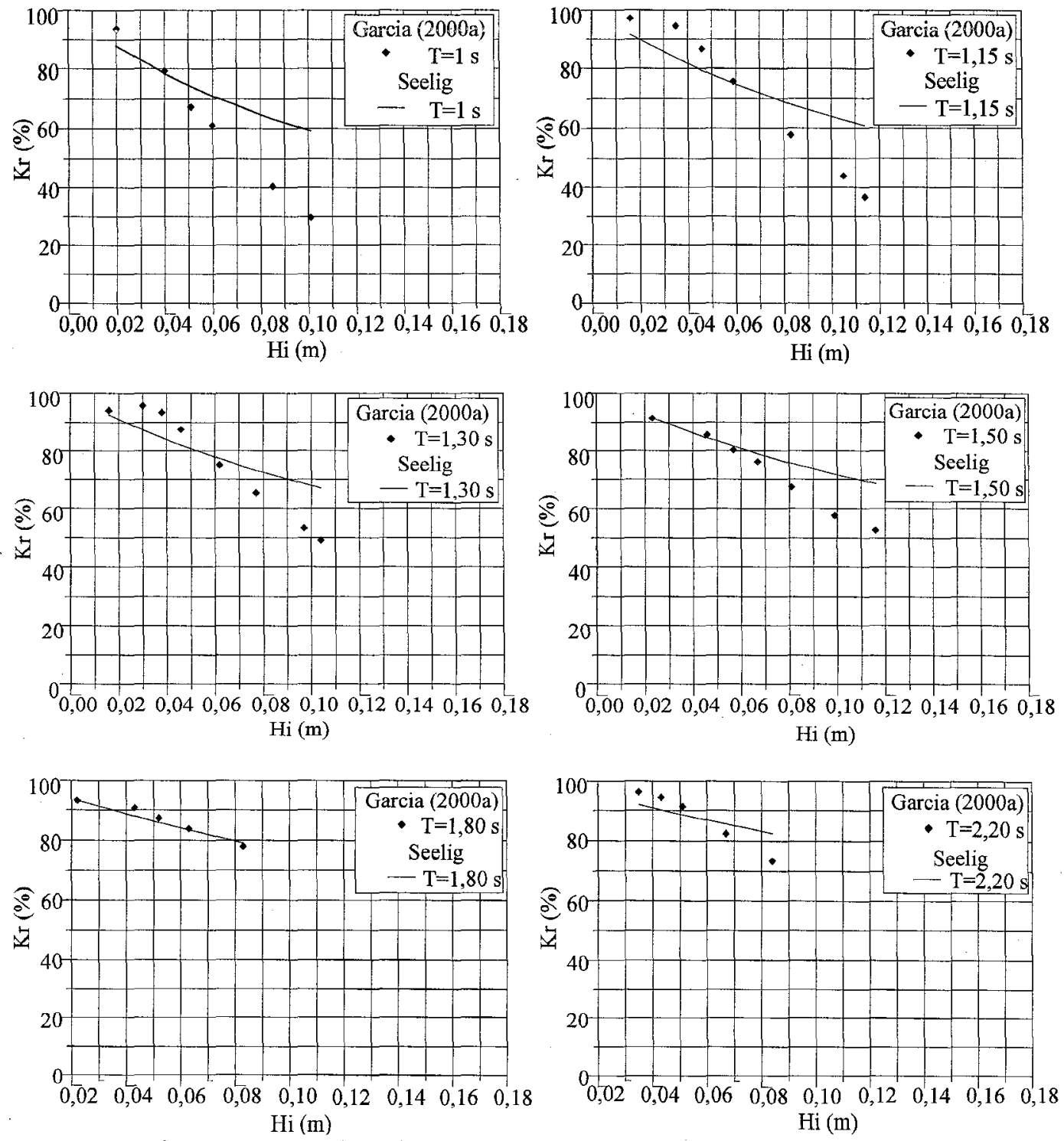

Figure 4 : Courbes de Seelig et résultats expérimentaux de $\mathrm{Kr}$ Comparison between Seelig curves and experimental results 
5. Formulations semi-empiriques proposées pour le calcul du coefficient de réflexion Kr

Nos résultats expérimentaux s'accordent sur la tendance d'une façon satisfaisante avec les résultats obtenus par Seelig. Il y a toutefois un écart important entre nos résultats expérimentaux du coefficient de réflexion et les résultats qu'il propose. En effet, comme nous l'avons déjà cité, le calcul de la réflexion reste un phénomène moins étudié que la transmission et les formulations théoriques que se trouvent dans la bibliographie restent assez limitées.

Il nous a paru intéressant à partir de nos données expérimentales, de proposer des formulations semi-empiriques concernant le calcul du coefficient de réflexion sur les digues à talus.

Sachant que la période de la houle est un facteur déterminant de la valeur du coefficient de réflexion, nous avons rassemblé les coefficients de réflexion obtenus au cours de notre expérience en deux groupes, un groupe pour les ondes courtes et l'autre pour les ondes longues.

Pour définir un domaine d'application de nos équations, nous avons choisi comme paramètre de référence le rapport $\mathrm{X} / \mathrm{L}$, avec $\mathrm{X}$ : avancée du talus sous marin, (voir figure 5).

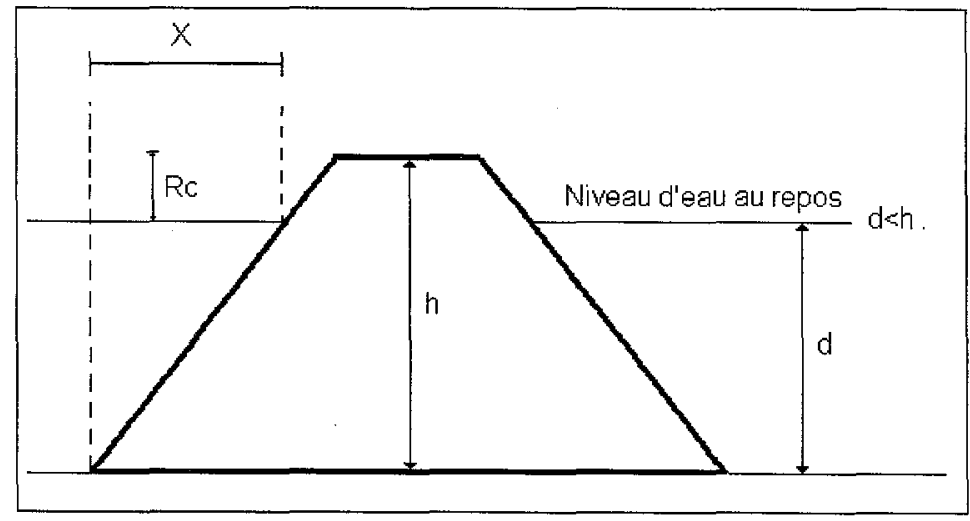

Figure 5 : Paramètres de référence - Parameters of reference

Les principaux facteurs qui ont une influence sur le coefficient de réflexion pour les structures à talus imperméable sont la profondeur relative $\mathrm{d} / \mathrm{h}$ et la période de la houle T. Nous proposons deux formulations issues d'une régression de type logarithmique où le coefficient de réflexion $\mathrm{Kr}$ est fonction de la cote d'arase de la digue Rc et de l'amplitude de la houle incidente Hi. Le tableau 2 présente les formulations données pour le calcul de $\mathrm{Kr}$.

Les courbes ajustées donnant les formulations 1 et 2 ont été rapportées sur les figures $6 \mathrm{a}$ et $\mathrm{b}$. 


\begin{tabular}{|c|c|c|}
\hline Type de houle & Courtes (formulation 1) & Longues (formulation 2) \\
\hline $\begin{array}{c}\text { Intervalle } \\
\text { d'application }\end{array}$ & $0,17<\frac{X}{L}<0,33$ & $0,11<\frac{X}{L}<0,17$ \\
\hline Formulation & $K r=0,739+0,299 \log \frac{R c}{H i}$ & $K r=0,893+0,158 \log \frac{R c}{H i}$ \\
\hline $\mathrm{R}^{2}$ & 0,76 & 0,59 \\
\hline
\end{tabular}

Tableau 2: Formulations proposées pour le calcul de $\mathrm{Kr}$, cas de la digue imperméable - Equations proposed by Garcia (2000a)

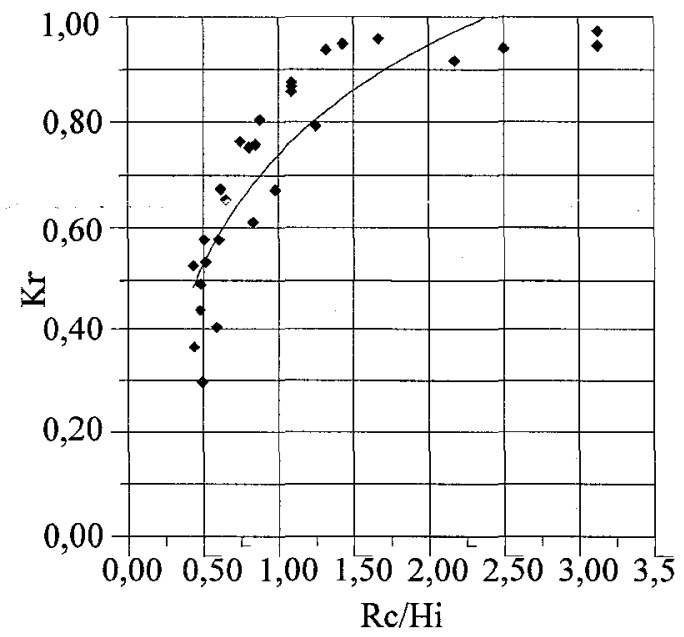

a) Formulation 1

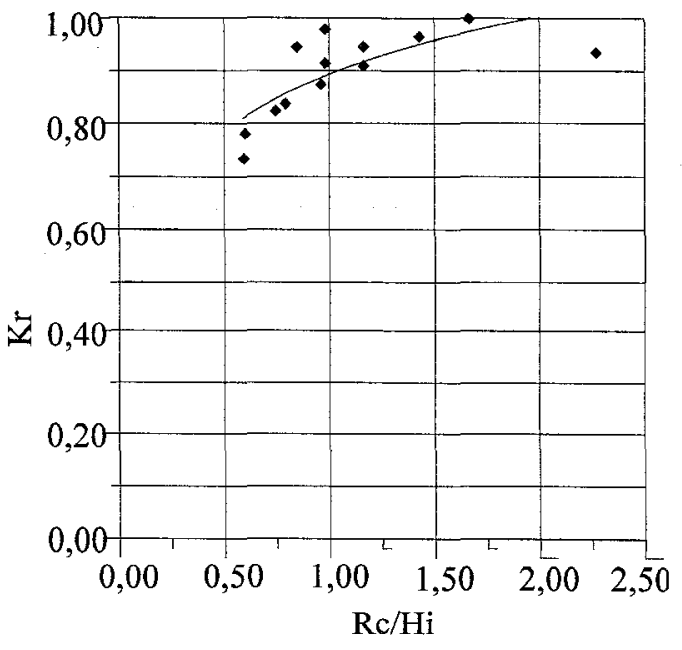

b) Formulation 2

Figure 6 : Courbes ajustées pour l'obtention des formulations

Fitting curves for obtaining proposed equations

\section{Conclusions}

La période de la houle joue un rôle fondamental pour le calcul de $\mathrm{Kr}$. La comparaison de nos résultats expérimentaux avec ceux obtenus par Seelig, montrent une assez bonne cohérence. Les formulations semi-empiriques proposées ne doivent en aucun cas être considérées comme une règle de l'art en matière de conception d'ouvrages. Elles ont pour ambition plus modeste de fournir des orientations en vue de l'élaboration d'une méthode plus précise pour le calcul du coefficient de réflexion. L'amélioration des formulations du coefficient de réflexion en faisant intervenir d'autres variables est suggérée pour l'obtention de formulation plus précises.

\section{Références Bibliographiques}

CHEVALIER C., 2000: "Action de la houle sur un cylindre immergé en zone côtière", $\mathrm{PhD}$ thesis, Université de Caen, $170 \mathrm{p}$. 
GARCIA-GOVEA M.C., 2000 a: "Etude experimentale de l'action de la houle sur les digues semi-submersibles", PhD thesis, Université de Caen Basse Normandie, France, $279 \mathrm{p}$.

GARCIA-GOVEA M. C., LEVACHER D., CAMINADE D., 2000 b: "Etude en laboratoire d'un amortisseur de houle constitué d'une plage parabolique et de deux plaques verticales perforées", VI ${ }^{\text {èmes }}$ Journées Nationales Génie Civil Génie Côtier, Caen, Centre Francais du Litoral, France, pp.91-100.

MANSARD E., FUNKE E., 1980: "The measurement of incident and reflected spectra using a least squares method", Coastal Engineering, Proceedings of 17 th Coastal Engineering Conference, Sydney Australia. pp. 154-172.

PILARCZYK 1996: "Offshore breakwaters and shore evolution control", A.A. Balkema (1996), $560 \mathrm{p}$.

ROUSSET J., BELORGEY M, 1998: "Analyse des variations de pressions générées par la houle sur la digue jarlan du Port de Dieppe", V ${ }^{\text {ièmes }}$ Journées Génie Civil-Génie Côtier, Toulon, France, pp.223-230.

SEELIG W., 1983: "Wave reflection from coastal structures", Proc. Conference of Coastal Structures, ASCE, Arlington, pp. 961-973.

\section{Notations}

\begin{tabular}{|c|c|c|c|}
\hline$a, b$ & $\begin{array}{l}=\text { coefficients liés à la formule de } \\
\text { Seelig; }\end{array}$ & $\mathrm{L}_{0}$ & $\begin{aligned}= & \text { longueur de la houle en eaux } \\
& \text { Profondes; }\end{aligned}$ \\
\hline $\mathrm{d}$ & $=$ profondeur d'eau; & $\mathrm{Rc}$ & $=$ cote d'arase; \\
\hline h & = hauteur de la digue; & $\mathrm{R}^{2}$ & $=$ coefficient de détermination; \\
\hline $\mathrm{Hi}$ & $=$ hauteur de la houle incidente; & $\mathrm{s}$ & $=$ sonde; \\
\hline $\mathrm{H}_{0}$ & $\begin{aligned}= & \text { hauteur de la houle en eaux } \\
& \text { Profondes; }\end{aligned}$ & $\mathrm{T}$ & $=$ période de la houle; \\
\hline $\mathrm{Hr}$ & $=$ hauteur de la houle réfléchie; & $X$ & = avancée du talus sous marin; \\
\hline $\mathrm{Kr}$ & $=$ coefficient de réflexion; & $\xi_{0}$ & $=$ Iribarren number; \\
\hline $\mathrm{L}$ & $=$ longueur de la houle; & $\xi_{\mathrm{P}}$ & $\begin{aligned}= & \text { Iribarren number correspondant à la } \\
& \text { fréquence pic d'un spectre. }\end{aligned}$ \\
\hline
\end{tabular}

